\title{
Differential phase shift keying in the research on the effects of type pattern of space optical communication system
}

\author{
Jiang Wenhao ${ }^{1}$, Jiang Lun ${ }^{2}$, Tong Shoufeng ${ }^{2}$, Li Xiaoyan ${ }^{2}$ \\ ${ }^{1}$ Changchun University Of Science and Technology, Optical Engineering. \\ Changchun,130000, China \\ ${ }^{2}$ Changchun University Of Science and Technology,Space laser communications technology \\ Key Laboratory of National Defense.Changchun,130000,China
}

Keywords: DPSK; CSRZ; atmospheric channel

\begin{abstract}
In the high-speed system of space optical communication, NRZ modulation on differential phase shift keying, 66\% duty cycle RZ modulation and 33\% duty cycle RZ modulation. By using the Optisystem optical communication software design of DPSK optical communication system based on communication rate in $40 \mathrm{~Gb} / \mathrm{s}$, the signal power is $-42 \mathrm{dBm}$, at a distance of 100 $\mathrm{km}$ for atmospheric channel $0.5 \mathrm{~dB} / \mathrm{km}$ transmission attenuation and then through the light of the noise coefficient of $3.2 \mathrm{~dB}$ preamplifier makes light signal gain after reaching $40 \mathrm{~dB}$, compares different modulation modes respectively discussed.
\end{abstract}

\section{Introduction}

DPSK modulation format (DPSK) is the use of before and after the relative carrier phase values of adjacent symbols to indicate modulation format digital information is a hot topic in recent years, optical transmission technology, in some experimental system performance superior performance. DPSK and off keying (OOK) modulation scheme compared at the same bit error rate, signal to noise ratio to lower $3 \mathrm{~dB}$, which can increase the transmission distance, optical power requirements and reducing devices.

In the free-space optical communications, high rate DPSK signal due to atmospheric disturbances impacts before and after the symbol of the signal light wave is basically the same, differential interference demodulating an optical signal can be overcome atmospheric turbulence disturbance optical signal phase information to a certain extent, So space optical communication with the DPSK modulation format than the other modulation format advantages, establish three patterns in atmospheric transport model based simulation software optisystem In this paper, we compare the final result of the atmospheric turbulence channel attenuation and other some attenuation affect the final receiving end signal pros and cons.

\section{Principle}

2.1 DPSK modulation and demodulation

DPSK modulation format also belong PSK format, based on the relative phase between adjacent symbols that carry digital information "1" or "0." Commonly referred to as DPSK, it is in phase 0 or $\pi$ represent information. which is:

$$
\Delta \varphi=\left\{\begin{array}{ll}
0 & \text { code } " 0 " \\
\pi & \text { code } " 1
\end{array} \quad \text { or } \Delta \phi= \begin{cases}0 & \text { code } " 1 \\
\pi & \text { code } " 0\end{cases}\right.
$$

Since the DPSK modulation format is not absolute phase data indicative of information, but information representing the relative phase difference, which avoids the PSK modulation format appears in inverted $\pi$ phenomenon. DPSK modulation format before encoding process should be pre-coded, and then loaded onto the modulator for encoding phase modulation.

DPSK signal data is stored in the phase difference of adjacent bits in the received signal at the receiving end to deal with interference demodulation, the phase information into intensity information. The main components of the demodulated terminal photodetectors balanced coherent 
demodulation and delay interferometer 1bit, then balanced detection, as shown in FIG.

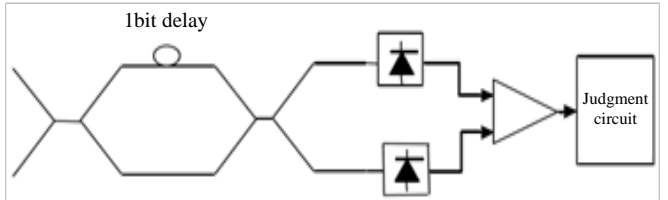

Figure 1. The main components of the demodulated end

2.2 DPSK pattern generation

DPSK transmitter configuration shown in FIG. The DFB laser transmitter connected by the latter consisting of two arms drive LiNbO3 Mach-Zehnder modulator. The first-stage code modulation data modulator, the second-stage modulator may be cut waveform, in order to reduce the push-pull driver Used chirp modulation.

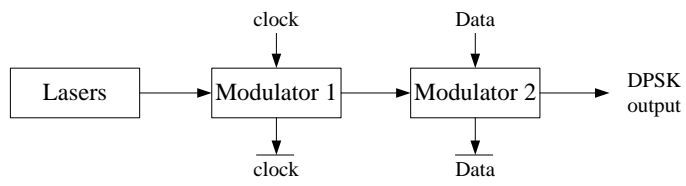

Figure 2. DPSK transmitter structure

Loading episode modulator that is used to implement the phase-code modulation data, the bias point is set at MZ-LN1 nulls transmission curve (Fig. 3 at $V_{\pi}$ ), a pair of differentially encoded NRZ data stream voltage drive, drive voltage peak value $2 V_{\pi}$, generate NRZ signal (Figure 4). A modulator for implementing the second stage cutting waveform, the bias placed MZ-LN2 transfer curve peak (at $2 V_{\pi}$ in Figure 3), a pair of half rate sinusoidal signal rate clock driver, the driving voltage peak value $2 V_{\pi}$, can generating a duty cycle of 33\% RZ-DPSK pulse sequence (Figure 4). Level modulator bias unchanged, secondary modulator bias point is set in the MZ-LN1 nulls transmission curve (Fig. 3 at $V_{\pi}$ ) obtained a duty cycle of 66\% CSRZ-DPSK signal (Figure 3).

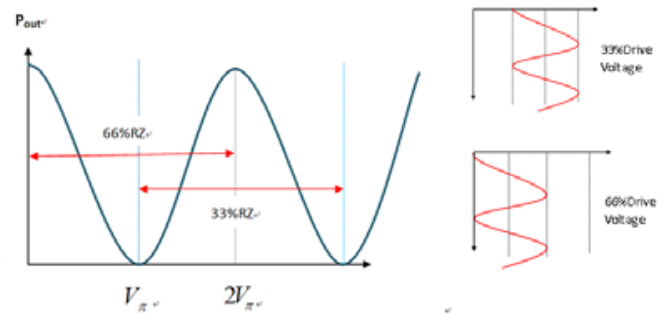

Figure 3. Mach-Zehnder modulator modulates curve (left) drive voltage (right)

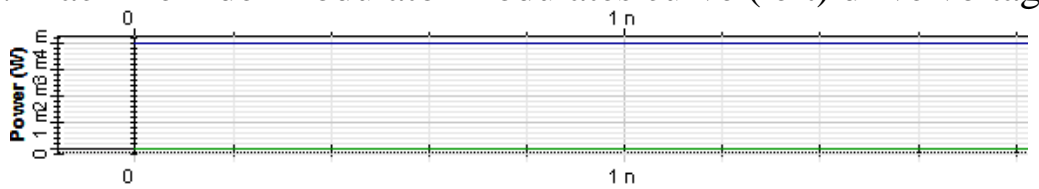

(a) NRZ-DPSK

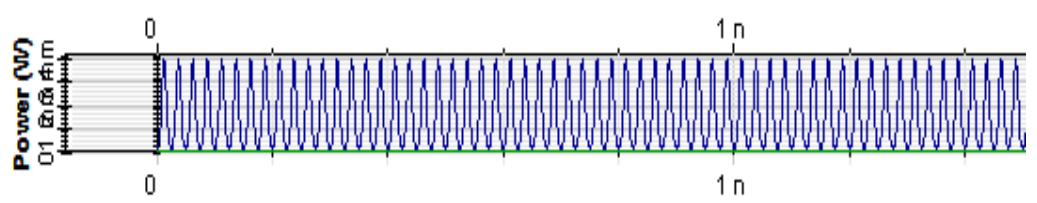

(b) $33 \% R Z-D P S K$

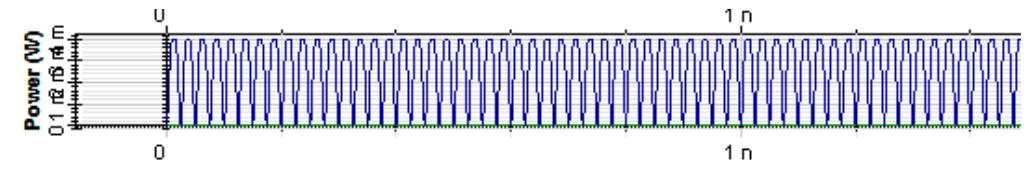

(c) $66 \%$ CSRZ-DPSK

\subsection{Amplifier gain and noise figure}

Figure 4. RZ-DPSK pulse sequence

After receiving the rear end of the optical EDFA preamplifier, in addition to the noise outside the signal itself, but also the introduction of spontaneous emission (ASE) noise, and signal light and the 
ASE (S-ASE) on the detector and the noise generated by the ASE-ASE. In addition to the EDFA optical signal is amplified input signal but also noise signals for this same amplification EDFA gain is defined as:

$$
G=\left(P_{\text {out }}-P_{\text {ASE }}\right) / P_{\text {in }}
$$

Wherein, $P_{\text {in }}$ and $P_{\text {out }}$ are the amplifier input and output signal power, and $P_{\text {ASE }}$ on behalf of EDFA ASE power output falls within the bandwidth of the signal light.

Noise EDFA noise figure used to measure, which defines the ratio of input and output optical signal-to-noise ratio of the light, the noise figure reflects the degree of signal to noise ratio by the optical preamplifier after falling.

$$
\mathrm{NF}=10 \lg \left(\frac{S N R_{\text {in }}}{S N R_{\text {out }}}\right)
$$

A more general noise figure (NF) expression as the formula (3), called quantum beat noise limit noise, also known as the signal light - spontaneous emission beat noise limit noise figure. Which represents the signal bandwidth $\Delta v, P_{A S E}$ represented within the signal bandwidth of ASE noise. Using this formula can know the noise figure and gain influence by ASE noise, ASE noise greater NF bigger.

$$
\mathrm{NF}=10 \lg \left(1 / \mathrm{G}+P_{\text {ASE }} / h v G \Delta v\right)
$$

\subsection{Atmospheric channel}

Atmospheric turbulence is a random air movement conditions, the refractive index changes due to random variation of the local temperature in the atmosphere, pressure brought, during transmission due to the distance between the transmitter and receiver, emitted optical power attenuation in the air channel, Since the received signal and the spread of the beam transmitter and receiver spread between geometric attenuation caused by other causes severely disrupted and weak. Channel transfer function as shown in equation (4) as follows:

$$
P_{\text {Received }}=P_{\text {Transmitted }} \frac{d_{R}^{2}}{\left(d_{T}+\theta R\right)^{2}} 10^{\frac{-\alpha B}{10}}
$$

\section{The simulation}

Optisystem7.0 using optical communication simulation software to build a communication system, the input of a pseudo-random bit sequence (PRBS) as a binary number sequence initial input rate of $10 \mathrm{~Gb} / \mathrm{s}$. Before modulation need to differential pre-coding that require delayed again after a NAND gate XOR. NRZ signal generated by the laser pulse generator into the NRZ modulation input MZM1 of, 1550nm wavelength narrow linewidth laser output after modulated by NRZ-DPSK signals. NRZ-DPSK signal output and then through 5Gb / s signal into the modulated sinusoidal clock input MZM2 at different points of the bias voltage and thus generate 33\% RZ-DPSK and 66\% CSRZ-DPSK signal.

Three modulation pattern signal through attenuator attenuation to $-42 \mathrm{dBm}$, then through the low noise EDFA amplification gain of $40 \mathrm{~dB}$ noise figure of $3.2 \mathrm{~dB}$. After narrowband filter smoothing 1bit delay interferometer phase coherent demodulation from the long end, destructive ends of the two outputs, and then use 4-order Bessel filter electric filter after balanced detection, the bandwidth is 0.8 times communication rate $(0.8 \mathrm{R})$, after analyzing the data recovery and observe the minimum BER eye diagrams. 


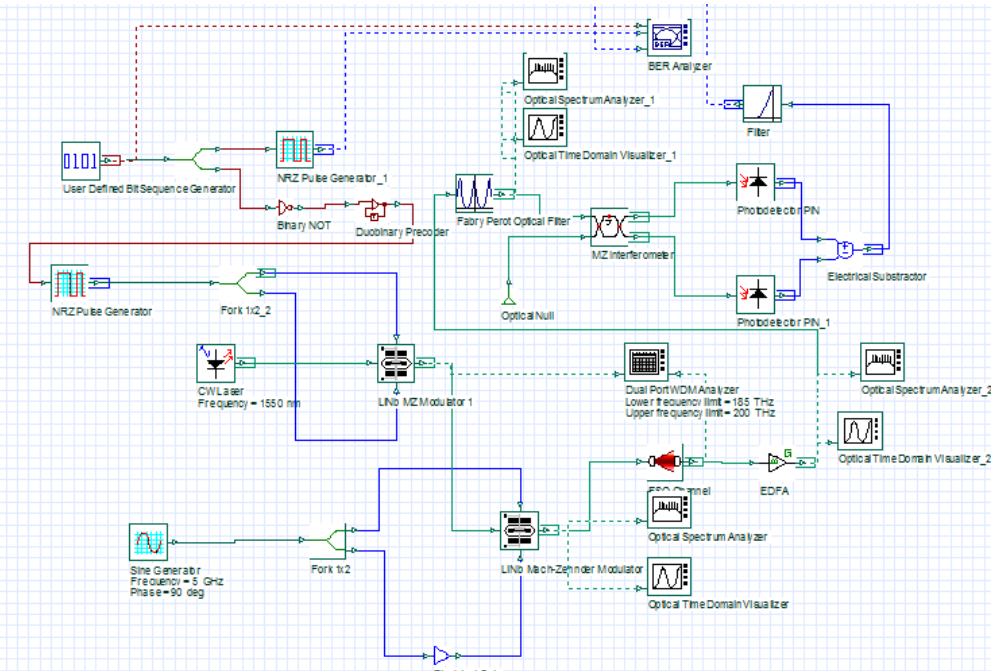

Figure 5. Simulation circuit

\section{Study results and analysis}

NRZ-DPSK, CSRZ-DPSK, 33\% RZ-DPSK modulation modes with three pattern optisystem software simulation results shown in Figure 5, Figure 6, we can see the signals transmitted through the atmosphere and then through the channel $100 \mathrm{Km}$ of EDFA optical after the pre-amplifier for signal amplification, the center frequency of the spectrum width CSRZ-DPSK signal is $26 \mathrm{GHz}$ and the side-lobe spectral power decrease speed decreases faster than the other two modulation mode, the center frequency spectral width RZ-DPSK signals is its 150G sidelobe spectral power fell faster than the slow CSRZ-DPSK, NRZ-DPSK center signal spectral width of the spectral power 20G side lobes decrease speed smoothly and RZ-DPSK similar.

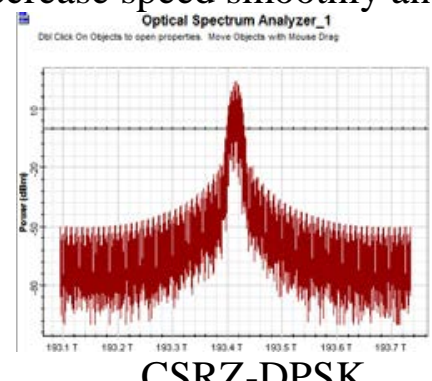

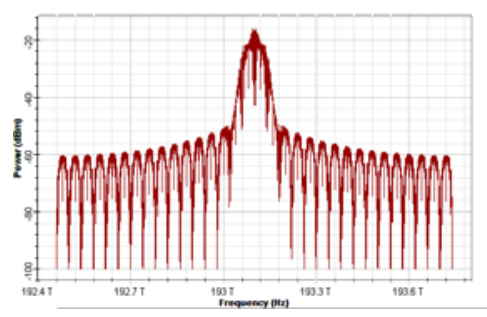

RZ-DPSK

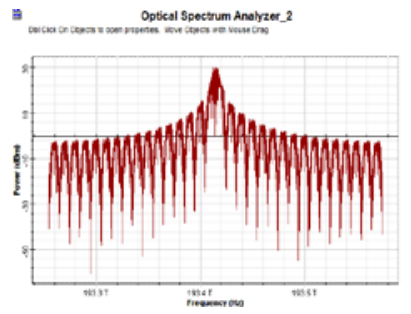

NRZ-DPSK

Figure 6. Three kinds of pattern modulation simulation results

The signal at the receiving end of three modulation modes eye diagram shown in Figure 7, wherein the CSRZ-DPSK eye opening degree of clarity and a better, 33\% RZ-DPSK eye opening degree is preferably clear but there are distortions, NRZ-DPSK eye chart more clearly, but less easily spread with a little noise of signal interference in the eye side.

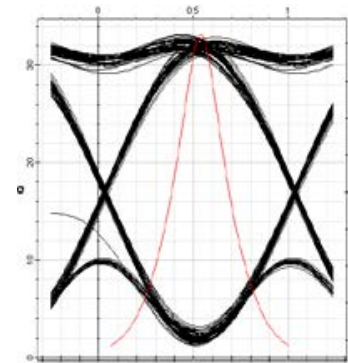

CSRZ-DPSK

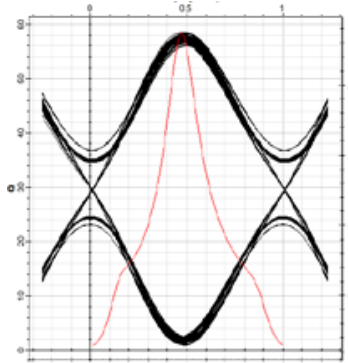

RZ-DPSK

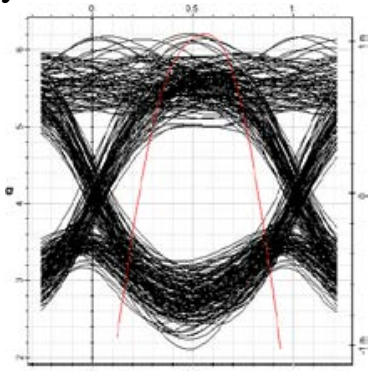

NRZ-DPSK

Figure 7. Eye of three modulation modes

\section{Summary}

For the impact of high-speed optical communication system space atmospheric turbulence 
channel is the most important factor in the overall system, in this paper we use the DPSK optisystem software simulation space optical communication system based on high-speed transmission in three signal modulation mode comparison, we can see that the spectral width of NRZ-DPSK minimum, eye opening more clear, but the noise signal interference, the spectral side lobes fall flat; CSRZ-DPSK spectral width of the second, the receiver signal eye clear and noise small sidelobe spectrum decreased rapidly; and RZ-DPSK large spectral width, DPSK eye clear but there are obvious distortion, spectral sidelobes fall flat. In summary narrow spectrum CSRZ-DPSK modulation mode center, strong anti-interference than the other two modulation schemes for high speed compared to more space optical communications systems, this paper only stay at the theoretical level simulation, and more specific method should further validation and exploration in the experiment.

\section{Reference}

[1] Feng Xie, Zhenhong YU, Optisystem of dispersion compensation fiber optic transmission systems for high-speed. Chinese Journal of Spectroscopy Laboratory, 2013, 11, Vol. 30, No. 6.

[2] Yang Xu, Song Lu, Wang Wei, Wang Shengda. Research and Simulation on Modulation Techniques in High-speed Optical Transmission System. Semiconductor Optoelctronics. 2011, 12, Vol. 32, No. 6.

[3] Xue Cao. Optimization of dispersion compensation in optical fiber communication systems. Laser Technology, 2014, 1(38): 1-2.

[4] GUWY, WENH, YUS, et al. WDM ultra-long optical transmission technologies Beijing: Beijing University of Post Telecommunication Press, 2010: 110-122 ( in Chinese) .

[5] Yanhua Sun, Zhe Wang, Zhanjun Jiang. Simulation and analysis of performance for 40Gb/s single channel optical fiber transmission system. Optical transmission, 2012, Vol.3. 